\title{
Generation of Periodic Micro- and Nano-structures by Parameter- Controlled Three-beam Laser Interference Technique
}

\author{
Stefan Beckemper ${ }^{* 1}$, Jintang Huang ${ }^{1,2}$, Arnold Gillner ${ }^{1}$ and Keyi Wang ${ }^{2}$ \\ ${ }^{* 1}$ Fraunhofer Institute for Laser Technology ILT, 52074 Aachen, Germany \\ E-mail: Stefan.beckemper@ilt.fraunhofer.de \\ 2 Department of Precision Machinery and Precision Instrumentation, University of Science and \\ Technology of China, Hefei 230027, China
}

\begin{abstract}
Periodic micro and nano structures are required for a variety of different products in micro optics, semiconductors and products with functional surfaces. Today the common approach is standard lithography with numerous processing steps including masking imaging, resist development and subsequent etching. Laser interference structuring is an appropriate solution to provide a manufacturing technology which is able to process polymers as well as semiconductors and metals in a single processing step without any subsequent etching. Based on the theory of interference superposition, the influence of different parameters is theoretically expressed and simulated. In this paper the influence of the phase and the polarization of the interfering beams upon the intensity distribution of the electrical fields of the three-beams are theoretically analyzed and experimentally demonstrated. It is demonstrated that in case of coplanar three-beam interference, the phase of the interfering beams has strong influence upon the intensity patterns of the interfering field. However, in case of non coplanar three-beam interference, the phase of the interfering beams has no influence upon the intensity patterns it just causes a lateral motion of the intensity pattern. By controlling the polarization arrangements of the laser beams, different intensity patterns, like circular holes, circular bumps or rectangular bumps can be generated. The demonstrated experiments about structuring on positive photoresist ma-P 1275 by laser ablation verify the simulated prediction very well. DOI:10.2961/jlmn.2011.01.0011
\end{abstract}

Keywords: Laser Ablation, Precision Micro Fabrication, Three-Beam Interference Technique, Nano-Structures

\section{Introduction}

As an excellent manufacturing technology, surface texturing has attracted increasing interest. Designed and welladdressed surface textures improve the performance of the surface remarkably, like friction, [1] water repellency [2] and adhesion [3]. Many methods like self-assembly, rapid prototyping, direct writing assembly, etching technique and holographic interference have been developed to pattern surfaces [4]. Among these methods, laser surface texturing (LST) is probably the most advanced one with respect to flexibility in rapid manufacturing. Moreover, compared with other techniques, LST provides excellent control of shape and size of the micro structures, which allows realization of optimum designs.

To produce patterned structures on material surfaces with LST technique, the laser beam position relative to the surface has to be manipulated. There are three main techniques for controlling the laser beam to create the desired patterns: direct beam movement, scanning, and holographic interference [5]. Holographic interference is a proven effective and inexpensive method for rapid fabrication of large area periodic structures in high resolution [6]. It is reported that by using four umbrella-like arranged beams interference, total 14 Bravais lattices including 10 threedimensional microstructures can be produced [7]. The conventional techniques show that interference with two or more coplanar beams will induce one-dimensional line structures and several symmetrically placed interfering beams will give rise to two-dimensional structures in the interfering area. A specific setup with a fixed beam alignment can only fabricate one specific structure. In order to obtain different structures, the optics alignment has to be changed. Recently, several groups have attempted to use phase manipulated multi-beam interference to fabricate different textures on the surface of the work pieces [8-10]. It is also reported that polarization changings lead to different intensity distributions. The parameter controlled laser beam interference is becoming a powerful tool for laser surface texturing [11-12].

In this contribution, we present results produced by parameter controlled three-beam interference technique. Based on the theory of the interference superposition, the influence of the parameter of the interfering beams upon the intensity distribution is simulated. In the experiment, interference with phase controlled three coplanar laser beams is carried out to fabricate one-dimensional lines. The polarization controlled three-beam interference is constructed to address structures of different topologies on for example positive photoresist (ma-P 1275) samples. 


\section{Theory}

According to the interference theory, the intensity pattern of multi-beam interference is formed by the square of the electrical field distribution of the interfering beams [11]:

$I(r)=\sum_{i=1}^{n}\left|\vec{E}_{i}\right|^{2}+\sum_{i \neq j}^{n} \vec{E}_{i}^{*} \cdot \vec{E}_{j}$

where $\overrightarrow{\mathrm{E}}_{\mathrm{i}}=\mathrm{E}_{\mathrm{i} 0} \cdot \exp \left\{\mathrm{j}\left(\overrightarrow{\mathrm{k}}_{\mathrm{i}} \cdot \overrightarrow{\mathrm{r}}-\delta_{\mathrm{i}}\right)\right\} \cdot \overrightarrow{\mathrm{e}}_{\mathrm{i}}$ represents the complex amplitude of the ith interfering beam. $\overrightarrow{\mathrm{r}}=(\mathrm{x} \mathrm{y} \mathrm{z})^{-1}$ is the position vector in the interfering space. $\mathrm{E}_{\mathrm{i} 0}, \overrightarrow{\mathrm{k}}_{\mathrm{i}}, \delta_{\mathrm{i}}$ and $\overrightarrow{\mathrm{e}}_{\mathrm{i}}$ are the real amplitude of the $i$ th plane wave, wave vector, initial phase, and unit vector in its polarization direction.

Assuming that the intensity of the interfering beams is equal to each other, three parameters exist that can be varied to control the intensity distribution of the interfering field, $\overrightarrow{\mathrm{k}}_{\mathrm{i}}, \delta_{\mathrm{i}}$ and $\overrightarrow{\mathrm{e}}_{\mathrm{i}}$. By changing the wave vector $\overrightarrow{\mathrm{k}}_{\mathrm{i}}$, the geometry of the lattice unit cell in the interfering area is changed. For this method, the optical alignment is required to be changed. By changing the phase $\delta_{\mathrm{i}}$ and the polariza-

tion $\overrightarrow{\mathrm{e}}_{\mathrm{i}}$ of the beams, the intensity distribution within the unit cell is changed.

Figure 1 is a schematic configuration of parameter controlled three-beam interference with discrete devices. Three laser beams split from one laser are placed in the designed position for the objective structures.

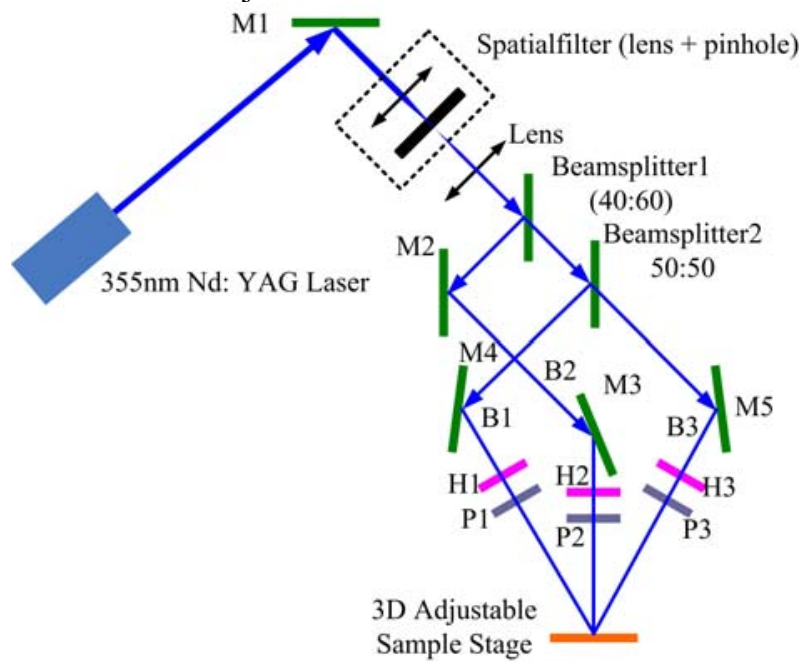

Figure 1: Schematic optical setup of three-beam interference setup M1, M2, M3, M4 and M5-totally reflective mirrors; H1, $\mathrm{H} 2$ and $\mathrm{H} 3$-half-wave plates; P1, P2 and P3- polarizers and two beamsplitters with different reflectivity (33:66 and 50:50)

\section{Coplanar three-beam interference}

Coplanar three-beam interference means that three interfering laser beams are placed in one plane. Figure 1 shows the arrangement of the three-beam interference setup. The superposition of three plane waves with the same intensity leads to a different intensity modulation compared to a two beam interference setup. Figure 2 shows a simulation of the intensity distribution depending on the amplitude of beam $\mathrm{B} 2$, where the amplitude of B1 and B3 is set to 1 . The blue curve shows the intensity distribution when the amplitude of $\mathrm{B} 2$ is set to zero. The intensity pattern here is equal to an intensity pattern of a two-beam interference setup. By increasing the intensity of B2 every other intensity peak will be suppressed. At an amplitude ratio of about 1.5 to 1 every other peak is almost totally suppressed. The periodicity of the distribution is doubled and the remaining peaks are obviously higher. With this method it is possible to generate deeper structures compared to two- beam interference technique.

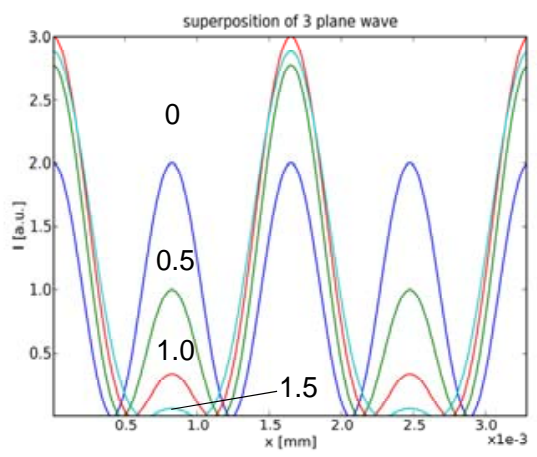

Figure 2: Intensity distribution along the projected line of the irradiating beams on the interfering plane with different settings of the amplitude of Beam B2. (The amplitude of Beam B1 and B2 is set to 1)

Figure 3 shows the intensity distribution along the projected line of the irradiating beams with different phase settings of the interfering beams. Figure 3(a) is the corresponding intensity with all beams are set to the same phase. Periodic sharp intensity peaks are formed along the projected line on the interfering plane. However, if the phase of one beam is shifted, the intensity distribution will be changed. As shown in Figure 3(b), where the phase shift $\Delta \Phi$ of Beam 2 with respect to the Beams B1 \& B3 is set by changing the length of the light path by moving the sample for example of about $85 \mu \mathrm{m}$. The intensity modulation is almost distorted. The maximum of the intensity is much lower than that of Figure 3(a), which means the intensity contrast of the interfering field is much weaker.

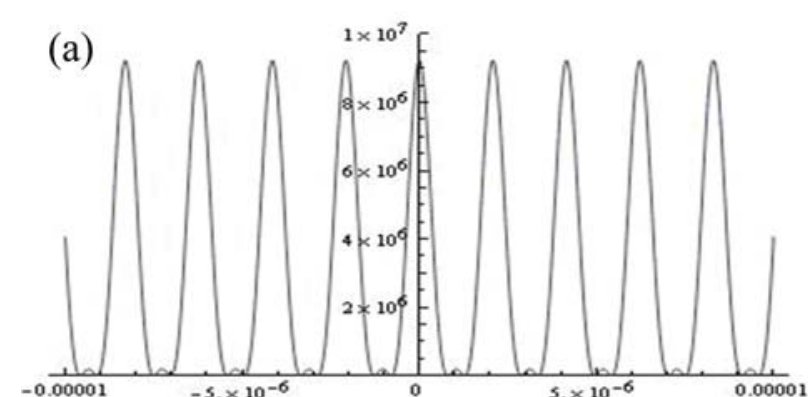

(b)

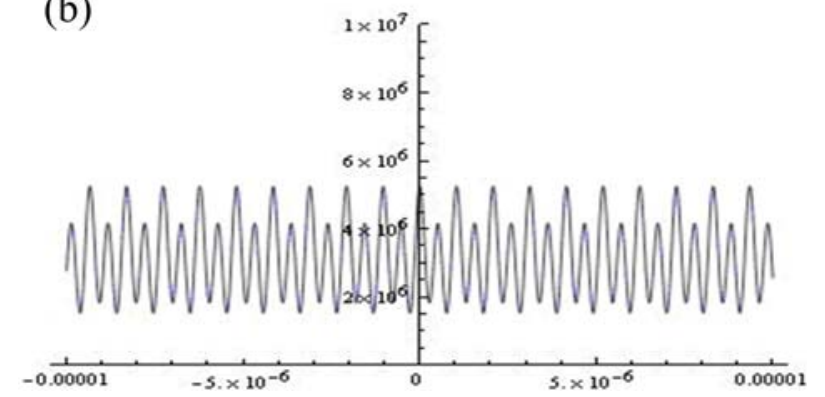


Figure 3: Calculated intensity distribution of the interfering field of three coplanar beams interference with different phase settings of the irradiating beams. (Phase settings: a$(0,0,0) ; \mathrm{b}-(0, \Delta \Phi, 0))$

The disadvantage of this technique is the phasesensitivity of the arrangement. Only in the optimal constellation of the three beams regarding to each other and the position of the sample an optimal intensity distribution will be obtained. Figure 4 shows a SEM image of microstructures in positive photoresist ma-P 1275 layer generated by the ablation via the coplanar three beam interference field with two different settings of the phase. Figure 4 (a) shows periodic lines (grooves) with the periodicity of about $3 \mu \mathrm{m}$. The phases of the interfering beams are all set to 0 , this is the optimal component alignment. Sharp lines with the size of about $600 \mathrm{~nm}$ are formed. Figure 4 (b) shows a SEM of the microstructure with a phase shift $\Delta \Phi$ of Beam 2 with respect to Beam $1 \& 3$. Periodic lines with the periodicity of about $800 \mathrm{~nm}$ are formed.

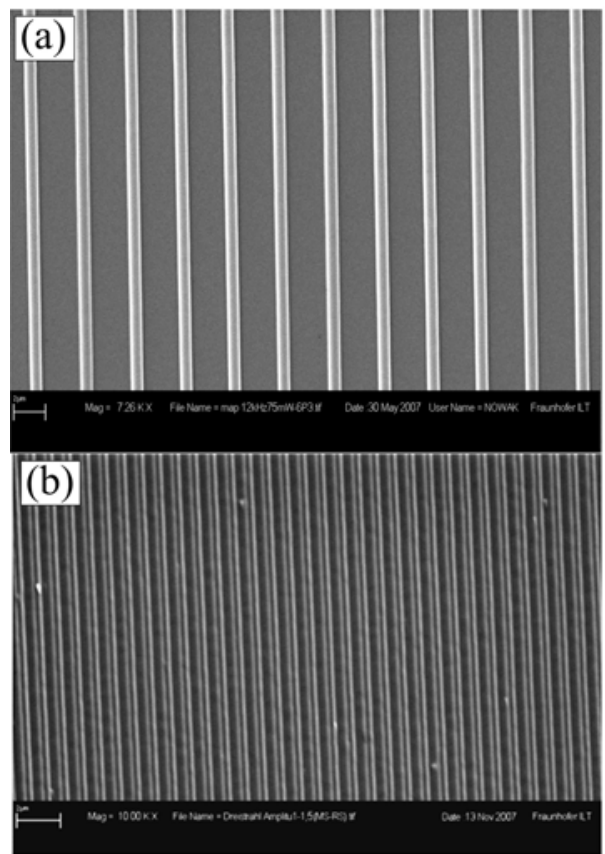

Figure 4: SEM images of microstructures fabricated by coplanar three-beam interference with different phase settings of the irradiating beams. (Phase setting: a- $(0,0,0)$; b$(0, \Delta \Phi, 0))$

\section{Non-coplanar three-beam interference}

As the upper demonstration, coplanar three-beam interference generates only one-dimensional gratings on the surface of the recording medium. To produce twodimensional structures, the three interfering beams should not be placed in one plane. For the symmetry of the produced structures, the non-coplanar beams are placed symmetrically around the normal of the sample surface.

In this section, we will analyze the influence of the parameters of the interfering beams upon the shape of the generated structures.

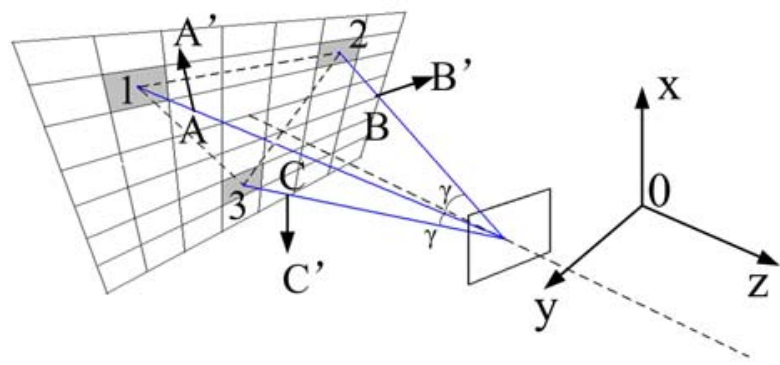

Figure 5: 3-dimensional laser beam arrangement of noncoplanar three-beam interference technique (AA', BB' and $\mathrm{CC}^{\prime}$ are in the planes of P-polarization of each beam)

\subsection{Phase influence}

For more than three-beam interference, the phase of the interfering beams has vastly influence upon the intensity distribution in the overlapping area [8-10]. However, at three-beam interference, arranged in the 3-dimensional way as shown in figure 5 , a phase shift will not destroy the shape of the intensity pattern, only moving the pattern in the overlapping plane [13]. Figure 6 shows pictures of the intensity pattern of phase-shifted three-beam interference. The wavelength of the interfering beams is set to $355 \mathrm{~nm}$, and all beams are set to polarize in the same direction. Figure $6 \mathrm{~B}$ shows the pattern when the phase of beam 3 is shifted by $90^{\circ}$. Compared with Figure $6 \mathrm{~A}$, where the phase of all beams is set to 0 , the shape of the pattern in Figure $6 \mathrm{~B}$ is the same as the pattern Figure 6A, but moves in quarter periodicity in the direction of the projected line of beam B3 on the image plane. Similarly, in Figure 6C, where the phase of beam $\mathrm{B} 2$ is set to $90^{\circ}$, the pattern seems to be the resultant pattern of Figure 6A moving in the direction of the projected line of beam $\mathrm{B} 2$ on the image plane. If the phase of beam B2 and B3 is set to $90^{\circ}$, the pattern of Figure $6 \mathrm{D}$ seems to be the moved pattern of Figure $6 \mathrm{~A}$ in the vector sum of the displacement in the directions of the projected lines of beam B2 and beam B3. The displacement value is calculated by $\mathrm{D}=\mathrm{d}^{*} \Phi / 2 \pi$, where $\Phi$ is the shifted phase and $d$ is the period of the pattern. The results indicate that the phase shift in three-beam interference will not change the shape of the intensity pattern but moves it in the image plane. This means that by carefully controlling the phase of the beams, phase controlled three-beam interference can be applied as a tool for optical micromanipulation, which has wide applications for example in the biology field. 

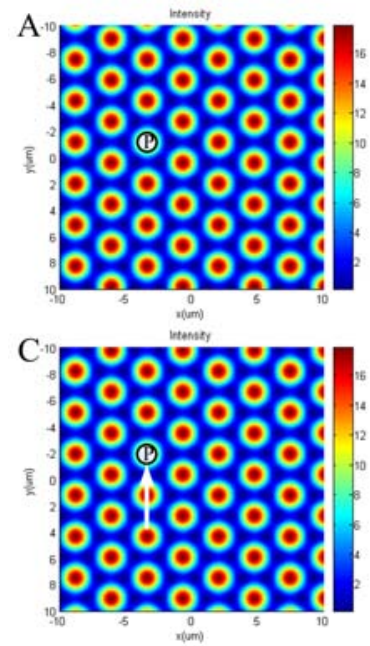
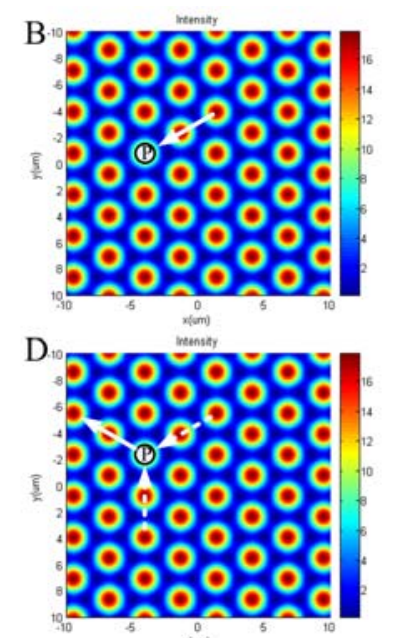

(1)
Figure 6: Intensity patterns of phase shifted three-beam interference (Phase setting: A- $\left(0^{\circ}, 0^{\circ}, 0^{\circ}\right)$; B- $\left(0^{\circ}, 0^{\circ}, 90^{\circ}\right)$; $\left.\mathrm{C}-\left(0^{\circ}, 90^{\circ}, 0^{\circ}\right) ; \mathrm{C}-\left(0^{\circ}, 90^{\circ}, 90^{\circ}\right)\right)$

\subsection{Polarization influence}

The phase shift of the interfering beams has no influence on the shape of the intensity pattern of the interfering field, yet the polarization of the interfering beams can be varied to control the pattern. For three-beam interference, polarization vectors are in the three-dimensional space, definition of the polarization is required. In this contribution, the definition of the polarization of the beams in paper [12] is adopted for the calculation and experimentation. As shown in Figure 7 , the beams are placed symmetrically around the $\mathrm{z}$ axis, making an interacting angle $\gamma$ to each other. Take a point (e.g. like A in Figure 5) on the corresponding beam and based on this point, make a vector (AA'), which is in the plane of P-polarization of each beam. Then the vector is defined as the $0^{\circ}$ value of the polarization of the corresponding beam. For common application cases, the interfering beams are set to the same polarization, then the corresponding polarization directions of the interfering beams are $\left(-120^{\circ}, 0^{\circ}, 120^{\circ}\right)$. Figure $7 \mathrm{~A}$ shows the intensity pattern of polarization arrangement $\left(-120^{\circ}, 0^{\circ}\right.$, $120^{\circ}$ ), where circular intensity bumps of hexagonal distribution are formed. As an alteration, the polarization arrangement is set to $\left(0^{\circ}, 0^{\circ}, 0^{\circ}\right)$, then the intensity pattern shown in Figure $7 \mathrm{~B}$ is obtained, where circular intensity cavities of hexagonal distribution are formed. Figure 7B seems the conversion of Figure 7A. When the polarization arrangement is set to $\left(-120^{\circ}, 45^{\circ}, 210^{\circ}\right)$, rectangular pattern are formed in the interfering field, as shown in Figure 7C.

For the experiment, as shown in Figure 1, a nanosecond diode pumped Nd:YAG laser with a wavelength of $355 \mathrm{~nm}$ was employed as light source. After being filtered and collimated, the laser beam is split by two beam splitters into three coherent beams for subsequent interfering fabrication. To control the polarization and the energy ratio of the interfering beams, one polarizer and one half-wave plate is placed in the path of each beam. The positive photoresist ma-P 1275 with the thickness of about $10 \mu \mathrm{m}$ produced by spin coating was prepared for the textures fabrication. The fluence of the beams is set to $60 \mathrm{~mJ} / \mathrm{cm}^{2}$ and the intersecting angle between the beams is set to $9.0^{\circ}$. To get sharp structures we used a single-shot strategy with a laser-pulse dura- tion of about $30 \mathrm{nsec}$. That means temperature-drifts or vibrations in the lab etc. make no contribution to that.

Figure $7 \mathrm{a}$ is the scanning electronic microscope image of the resultant structure corresponding to the polarization setting of Figure 7A. Circular cavities of hexagonal distribution are formed on the surface of the material. In Figure $7 \mathrm{~b}$, circular bumps of hexagonal distribution are formed, which matches the simulative predict in Figure 7B. Figure $7 \mathrm{c}$ is the corresponding structure of polarization arrangement of Figure 7C, where rectangular patterns are formed. Although the shapes of the patterns are different, the periodicities are all about $2.6 \mu \mathrm{m}$, showing good consistence with the theoretical value $2,612 \mu \mathrm{m}$ given by $\mathrm{d}=\lambda /(\sqrt{3} \sin (\gamma / 2))^{14}$

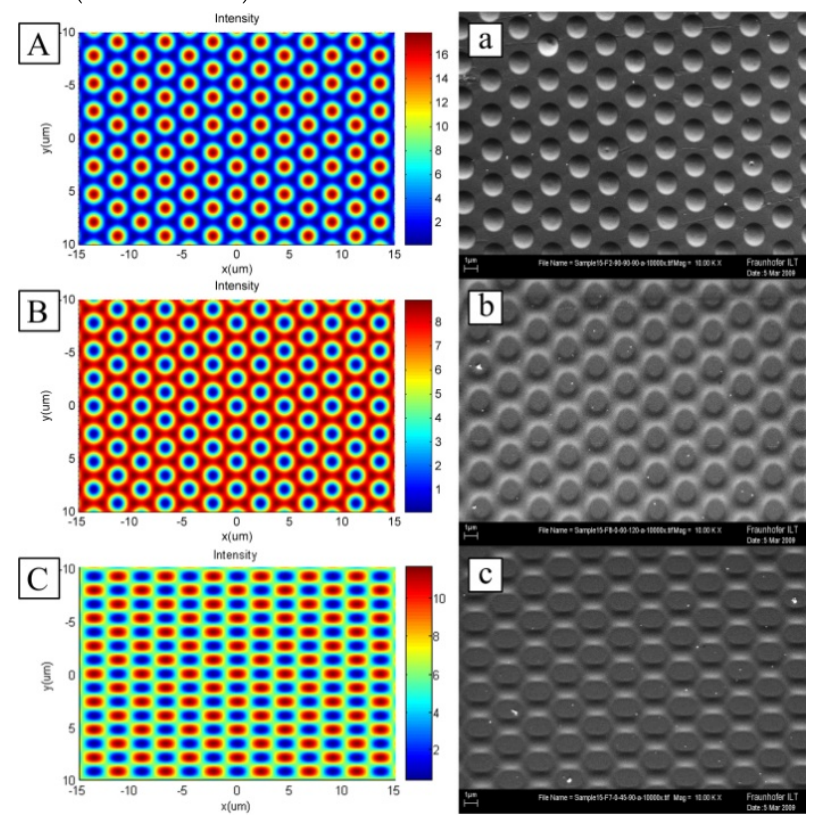

Figure 7: Calculated intensity distribution and SEM images of the structures fabricated by polarization controlled threebeam interference (Polarization arrangement-A,a- $\left(-120^{\circ}, 0^{\circ}\right.$, $\left.120^{\circ}\right)$; B,b- $\left(0^{\circ}, 0^{\circ}, 0^{\circ}\right)$; C,c- $\left.\left(-120^{\circ}, 45^{\circ}, 210^{\circ}\right)\right)$

\section{Conclusion}

Surface texturing with interference technique is demonstrated. Parameter controlled three-beam interference is utilized to address textures on the surface of polymer materials. Coplanar three-beam interference and non-coplanar three-beam interference are employed to fabricate onedimensional and two dimensional structures. The simulation and experimental results match to show that in coplanar three-beam interference, the phase of the interfering beams has strong influence on the intensity distribution of the interfering field. In non-coplanar three-beam interference, the phase shift of the interfering beam has no influence on the shape of the intensity pattern. However, by carefully changing the polarization of the interfering beam, circular micro-cavities, circular micro-bumps and rectangular bumps of hexagonal distribution can be produced on the sample surface without changing the optics alignment of the setup. Surface texturing with parameter controlled laser beams interference should have an broad application in many domains. 


\section{Acknowledgments and Appendixes}

This work is funded by the German Research Foundation DFG as part of the Cluster of Excellence "Integrative Production Technology for High-Wage Countries" and the National Natural Science Foundation of China (No. 50875251).

\section{References}

[1] R. A. Berthé, G. Westhoff, H. Bleckmann and S. N. Gorb, J Comp Physiol A Neuroethol Sens Neural Behav Physiol. 195 (2008) 311 (Journals)

[2] M. Callies and D. Quére, Soft Matter, 1(2005), 55. (Journals)

[3] A.K. Geim, S.V. Dubonos, I.V. Grigorieva, K.S. Novoselov, A.A. Zhukov and S.Y. Shapoval, Nature Materials, 2(2003), 461 (Journals)

[4] J. Moon and S. Yang, Polymer Reviews, 45(2005), 351 (Journals)

[5] M. Duarte, A. Lasagni, R. Giovanelli, J. Narciso, E. Louis and F. Mücklich, Advanced Engineering Materials, 10(2008), 554(Journals)

[6] M. Campbell, D. N. Sharp, M. T. Harrison, R. G. Denning, and A. J. Turberfield, Nature 404(2000), 53. (Journals)

[7] X.L. Yang, L.Z. Cai, Y.R. Wang and Q. Liu, Optics Communications, 224(2003), 243(Journals)

[8] T. Kondo, S. Matsuo, S. Juodkazis and H. Misawa, Appl. Phys. Lett. 79(2001), 725 (Journals)

[9] J. Klein-Wiele, K. Wielea and P. Simon, Appl. Phys. Lett., 83(2003), 4707 (Journals)

[10] Y. Yang, Q. Li and G. Wang, OPTICS EXPRESS, 16(2008), 11275(Journals)

[11] M.J. Escuti and G.P. Crawford, Opt. Eng., 43(2004), 1973 (Journals)

[12] F. Vita, D. E. Lucchetta, R. Castagna, L. Criante, and F. Simoni, Appl. Phys. Lett., 91(2007), 103114 (Journals)

[13] X.S. Xie, M. Li, J. Guo, B. Liang, Z.X. Wang, A. Sinistkii, Y. Xiang and J.Y. Zhou, OPTICS EXPRESS, 15(2007), 7032(Journals)

[14] L.Cai, X. Yang and Y. Wang, Journal of modern optics, 49(2002), 1663 (Journals)

(Received: June 07, 2010, Accepted: February 02, 2011) 